\section{Factores demográficos y comorbilidades asociadas a severidad de COVID-19 en un hospital chileno: el rol clave del nivel socioeconómico}

\author{
GASPAR DOMÍNGUEZ $Z^{1, a}$, CATALINA GARRIDO2,a,c, \\ MARÍA CORNEJO ${ }^{3, a, c}$, KAREN DANKE ${ }^{\mathrm{b}, \mathrm{d}}$, MIRTA ACUÑA ${ }^{4,5, \mathrm{a}}$
}

\section{Comorbidities and demographic factors associated with severe COVID-19 outcomes in a Chilean hospital}

Background: Several risk factors are associated with COVID-19 severity and death, such as advanced age, male sex, and the presence of comorbidities. Aim: To study the effect of these risk factors and socioeconomic variables on the outcome of Chilean patients admitted with COVID-19 to a Chilean public hospital. Material and Methods: Review of medical records of patients admitted to a Chilean public hospital with a positive PCR test for COVID-19, Chile from March to June 2020. The outcome variable was severity (ICU admission or death). The exposure variables were age, sex, socioeconomic level, and comorbidities. A multivariable logistic regression analysis was performed. Results: Of 1,141 confirmed cases, 266 cases had a severe evolution (23.3\%), including 147 deaths (fatality 12.9\%). Advanced age and low socioeconomic status were the variables most strongly associated with severity. An age of 80 years or over had an odds ratio $(O R)=11.1$ [ 95\% confidence intervals (CI) 5.22-23.53]. The OR [95\% CI] for a low socioeconomic level was 3.1 [1.1-8.5]. The figure for male sex was 2.13 [1.5-3.0], for chronic kidney disease was 2.65 [1.49-4.73], for obesity was 2.36 [1.65-3.39], and for diabetes 1.78 [1.22-2.61]. No significant association with severity was found for high blood pressure, chronic pulmonary disease, cardiovascular disease, or smoking. Conclusions: Following age, a low socioeconomic level was the factor with the higher association with a poor outcome or severe evolution of COVID-19.

(Rev Med Chile 2021; 149: 1141-1149)

Key words: COVID-19; Critical Care; Epidemiology; Social Determinants of Health; Mortality.

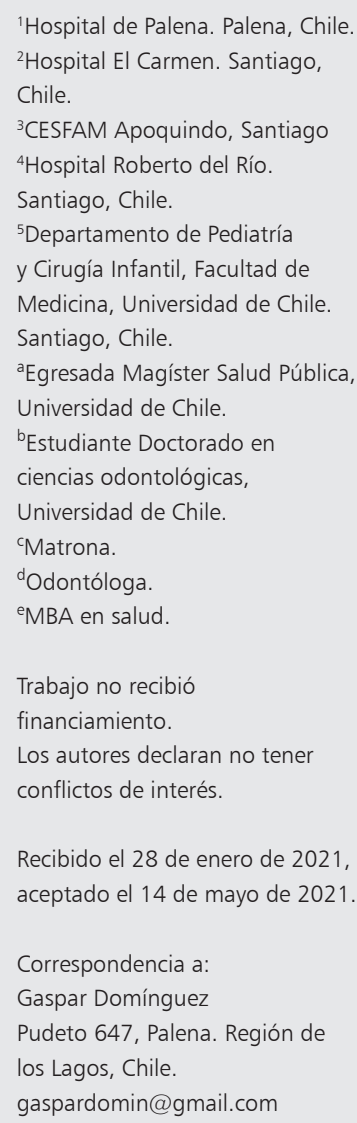

Recibido el 28 de enero de 2021 , aceptado el 14 de mayo de 2021

Correspondencia a:

Gaspar Domínguez

Pudeto 647, Palena. Región de

los Lagos, Chile.

gaspardomin@gmail.com

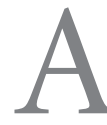
más de un año de los primeros casos de neumonía grave reportados en China, la enfermedad por SARS-CoV-2 ha tenido consecuencias devastadoras en todo el mundo. En Chile, al 2 de mayo de 2021 se han informado un total 768.671 casos acumulados (confirmados y probables) y 34.001 fallecidos $^{1}$, además de importantes secuelas sociales y económicas. La enfermedad por coronavirus 19 (COVID-19 por sus siglas en inglés) presenta gran variabilidad clínica que va desde casos asintomáticos hasta condiciones críticas que pueden llevar a la muerte ${ }^{2}$. Se ha descrito que la mayoría de los casos $(81 \%)$ se presentan como infecciones leves, aproxi- 
madamente $14 \%$ requiere hospitalización y $5 \%$ requiere manejo en unidades de cuidados intensivos $(\mathrm{UCI})^{3}$. En este último grupo se han descrito diversos factores demográficos y condiciones de salud preexistentes asociados con la gravedad y la muerte, en particular la edad, el sexo masculino ${ }^{4-8}$, bajo nivel socioeconómico 9 , y la presencia de enfermedades crónicas como enfermedades cardiovasculares, hipertensión, diabetes, enfermedades respiratorias, cáncer, obesidad, enfermedad renal crónica entre otras ${ }^{4-10}$. De estos factores, uno de los menos explorados hasta ahora es el rol del nivel socioeconómico. Estudios ecológicos nacionales e internacionales han mostrado la relevancia de esta variable en aumentar la carga de enfermedad y la mortalidad.

Conocer los factores asociados a gravedad de COVID-19 es esencial para desarrollar estrategias de mitigación y control en esta pandemia ${ }^{2}$, además permite al equipo de salud poner cuidado en aquellos pacientes con mayor probabilidad de complicaciones. De todas las muertes en el mundo, $25 \%$ se ha reportado solo en América del Sur ${ }^{13}$, sin embargo, los datos de estudios provenientes de esta región son escasos siendo subrepresentada en los metaanálisis que evalúan factores de riesgo para evolución severa, y muchos de ellos no consideran el nivel socioeconómico como variable en sus estimaciones $^{4-8}$, lo que podría generar confusión en los resultados ${ }^{14}$.

El objetivo de este estudio es analizar los factores demográficos y las comorbilidades asociadas con el resultado grave de COVID-19, incluyendo el nivel socioeconómico como un nuevo factor a explorar. Los resultados contribuirán a aumentar la representación de Sudamérica en el análisis del panorama global y entregarán elementos para direccionar la toma de decisiones en Chile con información local.

\section{Material y Método}

\section{Diseño del estudio}

Se realizó un estudio de cohorte retrospectivo con todos los pacientes COVID-19 del Hospital El Carmen (HEC) confirmados por reacción de polimerasa en cadena (PCR). El período de estudio comprende desde el 16 de marzo al 5 de junio de 2020, incluyendo hospitalizados y ambulatorios. El diseño de este estudio se ajusta a los postulados de la Declaración de Helsinki y fue aprobado por el Comité de Ética para la Investigación en Seres Humanos de la Facultad de Medicina de la Universidad de Chile.

\section{Variables}

La variable de resultado fue la severidad, definida como muerte o ingreso en la unidad de cuidados intensivos (UCI). Para efectos de esta variable, ingreso a UCI consideró el ingreso formal a dicha unidad o el manejo con un tubo orotraqueal fuera de la UCI (a veces en una sala de emergencias o camas modificadas fuera de la UCI). Las variables de exposición fueron datos demográficos: edad, sexo, nivel socioeconómico y presencia de comorbilidades: hipertensión, obesidad, diabetes, tabaquismo, enfermedad renal crónica (ERC), enfermedad pulmonar crónica y enfermedad cardiovascular.

\section{Fuente de datos}

Registros clínicos de cada caso confirmado mediante PCR. La muerte se obtuvo de la historia clínica electrónica (HCE). El registro de muerte luego del egreso se realizó con registro nacional de defunciones (Servicio de Registro Civil e Identificación de Chile) hasta tres meses después de la fecha de alta. La muerte por COVID-19 se determinó cuando al menos uno de los diagnósticos de defunción mencionados se designó explícitamente como COVID-19, COVID o SARS-CoV-2. Todos los datos fueron proporcionados por el Hospital El Carmen anonimizados previo al análisis.

Las variables de exposición edad, sexo y comorbilidades se determinaron mediante la historia clínica de los casos. La edad se categorizó en los siguientes grupos etarios, 15-39, 40-49, 50-59, $60-69,70-79$ y 80 o más. Se excluyeron los menores de 14 años debido a la baja prevalencia de comorbilidades. La variable socioeconómica se construyó de acuerdo con el tipo de afiliación al seguro de salud que tenía el paciente, generando tres categorías: baja, media y alta. En Chile, la evidencia muestra que el nivel socioeconómico puede estimarse por afiliación al seguro de salud. Aproximadamente $80 \%$ de la población está afiliada al sistema de seguro médico público denominado FONASA. ${ }^{15}$ Las personas pertenecientes a este seguro se agrupan en las secciones A, B, C y D según sus ingresos (que es proxy del NSE). La sección A corresponde a personas carentes de 
recursos o indigente, sin ingresos formales. Las secciones $\mathrm{B}, \mathrm{C}$ y D muestran ingresos crecientes y una mayor variabilidad ${ }^{16}$. De hecho los ingresos altos cuando son funcionarios de salud pública se les asigna $\mathrm{B}$, por lo que esta categoría es más heterogénea y podría incluir medios y altos (nunca bajo). El seguro de salud privado concentra a los usuarios con los ingresos más altos ${ }^{15}$. Por lo tanto, la categoría "baja" incluyó a los afiliados al seguro de salud público en tramo A, la categoría "media" incluyó las secciones B, C y D, y la categoría "alta" incluyó los casos afiliados al sistema ISAPRE.

Todos los casos sin información sobre seguro médico o comorbilidades y menores de 14 años fueron excluidos. Los datos duplicados por contar con más de una PCR en el laboratorio se consideró solo un dato. Cada una de las variables de comorbilidad se dicotomizaron como ausentes o presentes según la información presente en HEC, considerando las siguientes condiciones: hipertensión, obesidad, diabetes, ERC, enfermedad pulmonar crónica, enfermedad cardiovascular y fumadores activos. La enfermedad pulmonar incluyó enfermedad pulmonar obstructiva crónica, asma y fibrosis pulmonar. La enfermedad cardiovascular incluyó antecedentes de isquemia o infarto miocárdico, accidente cerebrovascular o enfermedad arterial periférica.

\section{Análisis estadístico}

Se realizó un análisis descriptivo de la muestra, indicando la prevalencia de ingreso en UCI y muerte para cada variable de exposición y categoría demográfica. Se realizó un análisis de regresión logística multivariable, con el resultado de severidad (medido como muerte e ingreso en UCI). El modelo incluyó sexo, tomando como referencia el sexo femenino; edad, tomando como referencia el grupo 40-49 y nivel socioeconómico, tomando como referencia el alto. Las comorbilidades fueron dicotómicas, utilizando la ausencia como referencia para el análisis. Se realizaron dos modelos de regresión logística multivariados, el primero ajustando por edad y sexo, y el segundo completamente ajustado, es decir todas las variables de exposición medidas fueron ajustadas. Ambos modelos se informan con intervalos de confianza de 95\%. Este modelo multivariado se utilizó para cuantificar los efectos independientes de cada variable de exposición después de ajustar los factores de confusión.

\section{Resultados}

\section{Participantes}

Durante el período estudiado hubo 1.243 casos de COVID-19 confirmados por PCR en el hospital de HEC. Se excluyeron 67 casos por tener 14 años o menos. Diecisiete fueron descartados debido a la falta de información del seguro médico. Uno fue excluido por no presentar información sobre comorbilidades. Se eliminaron 17 registros duplicados. Finalmente, se incluyeron para el análisis 1.141 casos confirmados (Figura 1). Todos los participantes estaban egresados en el momento del cierre del estudio y aquellos dados de alta a su domicilio se realizó un seguimiento de al menos tres meses para corroborar el estado de vivos o fallecidos.

\section{Datos descriptivos}

La muestra final estuvo formada por 54\% de mujeres (616 casos) y $46 \%$ de hombres, con edades comprendidas entre los 15 y los 102 años. La mediana de edad fue de 51 años (rango intercuartilico 33-64). El 8,94\% de la muestra se encontraba en el nivel socioeconómico bajo. Entre las comorbilidades estudiadas, la más frecuente fue la hipertensión con 32,52\% y la obesidad con

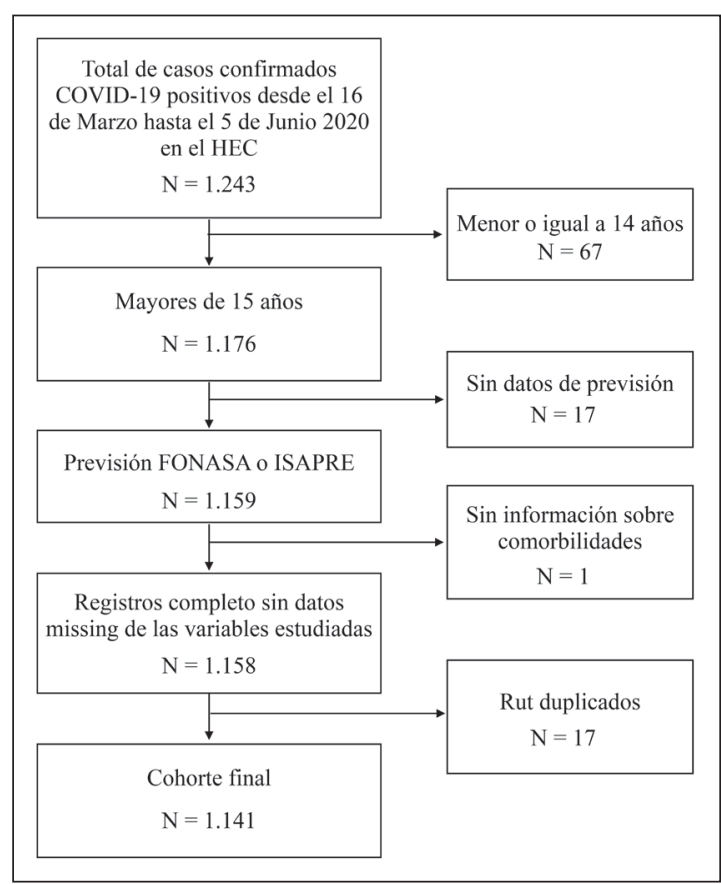

Figura 1. Diagrama de descripción de selección de la cohorte. 
$25,07 \%$. Las estadísticas descriptivas se presentan con más detalle en la Tabla 1, desagregadas por sexo y edad.

\section{Datos de resultados}

Del total de la muestra hubo 266 casos de evolución severa $(23,31 \%)$ incluyendo un total de 147 defunciones (letalidad 12,88\%). La Tabla 1 detalla el resultado para cada categoría de hombres, mujeres y ambos sexos (cuidados intensivos, muerte y severo, que incluye los dos anteriores).

Tabla 1. Tabla descriptiva de la cohorte para ambos sexos, hombres y mujeres por separado, del número de casos COVID-19 positivo confirmados por PCR, los casos que requirieron de manejo intensivo pero no murieron, los que murieron, y los casos severos considerados como manejo intensivo o muerte

\begin{tabular}{|c|c|c|c|c|c|}
\hline & & $\begin{array}{c}\text { Casos } \\
\text { positivos } \\
(n / \%)\end{array}$ & $\begin{array}{l}\text { Cuidados } \\
\text { intensivos } \\
(\mathbf{n} / \%)\end{array}$ & $\begin{array}{l}\text { Muerte } \\
\text { (n/\%) }\end{array}$ & $\begin{array}{l}\text { Casos } \\
\text { severos } \\
(\mathbf{n} / \%)\end{array}$ \\
\hline $\begin{array}{l}\text { Ambos sexos } \\
\text { Edad }\end{array}$ & $\begin{array}{l}\text { Todas las edades } \\
15-39 \\
40-49 \\
50-59 \\
60-69 \\
70-79 \\
>80\end{array}$ & $\begin{array}{l}1,141(100) \\
381(33,39) \\
160(14,02) \\
222(19,46) \\
203(17,79) \\
107 \quad(9,38) \\
68 \quad(5,96)\end{array}$ & $\begin{array}{l}119(10,43) \\
6(1,57) \\
18(11,25) \\
38(17,12) \\
36(17,73) \\
17(15,89) \\
4(5,88)\end{array}$ & $\begin{array}{ll}147 & (12,88) \\
4 & (1,05) \\
3 & (1,88) \\
22 & (9,91) \\
41 & (20,20) \\
37 & (34,58) \\
40 & (58,82)\end{array}$ & $\begin{array}{l}266(23,31) \\
10(2,62) \\
21(13,13) \\
60(27,03) \\
77(37,93) \\
54(50,47) \\
44(64,71)\end{array}$ \\
\hline $\begin{array}{l}\text { Nivel socio- } \\
\text { económico }\end{array}$ & $\begin{array}{l}\text { Alto } \\
\text { Medio } \\
\text { Bajo }\end{array}$ & $\begin{array}{l}110(9,64) \\
929(81,42) \\
102 \quad(8,94)\end{array}$ & $\begin{array}{cc}3 & (2,73) \\
99 & (10,66) \\
17 & (16,67)\end{array}$ & $\begin{array}{c}3(2,73) \\
131(14,10) \\
13(12,75)\end{array}$ & $\begin{array}{c}6(5,45) \\
230(24,76) \\
30(29,41)\end{array}$ \\
\hline Comorbilidades & $\begin{array}{l}\text { Obesidad } \\
\text { Hipertensión } \\
\text { Diabetes } \\
\text { Enfermedad renal crónica } \\
\text { Enfermedad pulmonar crónica } \\
\text { Enfermedad cardiovascular } \\
\text { Tabaquismo }\end{array}$ & $\begin{array}{c}286(25,07) \\
386(33,83) \\
250(21,91) \\
71(6,22) \\
66(5,78) \\
79(6,92) \\
115(10,08)\end{array}$ & $\begin{array}{l}61(21,33) \\
66(17,10) \\
47(18,80) \\
10(14,08) \\
11(16,67) \\
11(13,92) \\
14(12,17)\end{array}$ & $\begin{array}{l}64(22,38) \\
99(25,65) \\
70(28,00) \\
32(45,07) \\
19(28,79) \\
18(22,78) \\
12(10,43)\end{array}$ & $\begin{array}{l}125(43,71) \\
165(42,75) \\
117(46,80) \\
42(59,15) \\
30(45,45) \\
29(36,71) \\
26(22,61)\end{array}$ \\
\hline $\begin{array}{l}\text { Mujeres } \\
\text { Edad }\end{array}$ & $\begin{array}{l}\text { Todas las edades } \\
15-39 \\
40-49 \\
50-59 \\
60-69 \\
70-79 \\
>80\end{array}$ & $\begin{array}{c}616(54) \\
263(42,69) \\
83(13,47) \\
97(15,75) \\
82(13,31) \\
54(8,77) \\
37(6,01)\end{array}$ & $\begin{array}{cc}38 & (6,17) \\
2 & (0,76) \\
1 & (1,20) \\
15 & (15,46) \\
12 & (14,63) \\
7 & (12,96) \\
1 & (2,70)\end{array}$ & $\begin{array}{lc}63 & (10,23) \\
2 & (0,76) \\
0 \\
4 & (4,12) \\
14 & (17,07) \\
22 & (40,74) \\
21 & (56,76)\end{array}$ & $\begin{array}{cc}101 & (16,40) \\
4 & (1,52) \\
1 & (1,20) \\
19 & (19,59) \\
26 & (31,71) \\
29 & (53,70) \\
22 & (59,46)\end{array}$ \\
\hline $\begin{array}{l}\text { Nivel socio- } \\
\text { económico }\end{array}$ & $\begin{array}{l}\text { Alto } \\
\text { Medio } \\
\text { Bajo }\end{array}$ & $\begin{array}{c}62(10,06) \\
499(81,01) \\
55(8,93)\end{array}$ & $\begin{array}{cc}1 & (1,61) \\
33 & (6,61) \\
4 & (7,27)\end{array}$ & $\begin{array}{c}1(1,61) \\
55(11,02) \\
7(12,73)\end{array}$ & $\begin{array}{cc}2(3,23) \\
88(17,64) \\
11(20,00)\end{array}$ \\
\hline Comorbilidades & $\begin{array}{l}\text { Obesidad } \\
\text { Hipertensión } \\
\text { Diabetes } \\
\text { Enfermedad renal crónica } \\
\text { Enfermedad pulmonar crónica } \\
\text { Enfermedad cardiovascular } \\
\text { Tabaquismo }\end{array}$ & $\begin{array}{ll}151 & (24,51) \\
183 & (29,71) \\
128 & (20,78) \\
28 & (4,55) \\
39 & (6,33) \\
40 & (6,49) \\
54 & (8,60)\end{array}$ & $\begin{array}{l}22(14,57) \\
27(14,75) \\
20(15,63) \\
5(17,86) \\
4(10,26) \\
3 \quad(7,50) \\
1 \quad(1,85)\end{array}$ & $\begin{array}{l}26(17,22) \\
43(23,50) \\
32(25,00) \\
11(39,29) \\
13(33,33) \\
10(25) \\
5(9,26)\end{array}$ & $\begin{array}{l}48(31,79) \\
70(38,25) \\
52(40,63) \\
16(57,14) \\
17(43,59) \\
13(32,50) \\
6(11,11)\end{array}$ \\
\hline
\end{tabular}


Severidad de COVID-19: el rol clave del nivel socioeconómico - G. Domínguez et al

\begin{tabular}{|c|c|c|c|c|c|}
\hline & & $\begin{array}{c}\text { Casos } \\
\text { positivos } \\
(n / \%)\end{array}$ & $\begin{array}{l}\text { Cuidados } \\
\text { intensivos } \\
(n / \%)\end{array}$ & $\begin{array}{l}\text { Muerte } \\
\text { (n/\%) }\end{array}$ & $\begin{array}{c}\text { Casos } \\
\text { severos } \\
(\mathbf{n} / \%)\end{array}$ \\
\hline \multicolumn{6}{|c|}{$\begin{array}{l}\text { Hombres } \\
\text { Edad }\end{array}$} \\
\hline & Todas las edades & $525(46)$ & $81(15,43)$ & $84(16,00)$ & $165(31,43)$ \\
\hline & $15-39$ & $118(22,48)$ & $4(3,39)$ & $2(1,69)$ & $6 \quad(5,08)$ \\
\hline & $40-49$ & $77(14,67)$ & $17(22,08)$ & $3(3,90)$ & $20(25,97)$ \\
\hline & $50-59$ & $125(23,81)$ & $23(18,40)$ & $18(14,40)$ & $41(32,80)$ \\
\hline & $60-69$ & $121(23,05)$ & $24(19,83)$ & $27(22,31)$ & $51(42,15)$ \\
\hline & $70-79$ & $53(10,10)$ & $10(18,89)$ & $15(28,30)$ & $25(47,17)$ \\
\hline & $>80$ & $31 \quad(5,90)$ & $3(9,68)$ & $19(61,29)$ & $22(70,97)$ \\
\hline \multicolumn{6}{|c|}{ Nivel Socioeconómico } \\
\hline & Alto & $48 \quad(9,14)$ & $2(4,17)$ & $2(4,17)$ & $4(8,33)$ \\
\hline & Medio & $430(81,90)$ & $66(15,35)$ & $76(17,67)$ & $142(33,02)$ \\
\hline & Bajo & $47 \quad(8,95)$ & $13(27,66)$ & $6(12,77)$ & $19(40,43)$ \\
\hline \multicolumn{6}{|c|}{ Comorbilidades } \\
\hline & Obesidad & $135(25,71)$ & $39(28,89)$ & $38(28,15)$ & $77(57,04)$ \\
\hline & Hipertensión & $203(38,67)$ & $39(19,21)$ & $56(27,59)$ & $95(46,80)$ \\
\hline & Diabetes & $122(23,24)$ & $27(22,13)$ & $38(31,15)$ & $65(53,28)$ \\
\hline & Enfermedad renal crónica & $43(8,19)$ & $5(11,63)$ & $21(48,84)$ & $26(60,47)$ \\
\hline & Enfermedad pulmonar crónica & $27 \quad(5,14)$ & $7(25,93)$ & $6(22,22)$ & $13(48,15)$ \\
\hline & Enfermedad cardiovascular & $39(7,43)$ & $8(20,51)$ & $8(20,51)$ & $16(41,03)$ \\
\hline & Tabaquismo & $62(11,81)$ & $13(20,97)$ & $7(11,29)$ & $20(32,26)$ \\
\hline
\end{tabular}

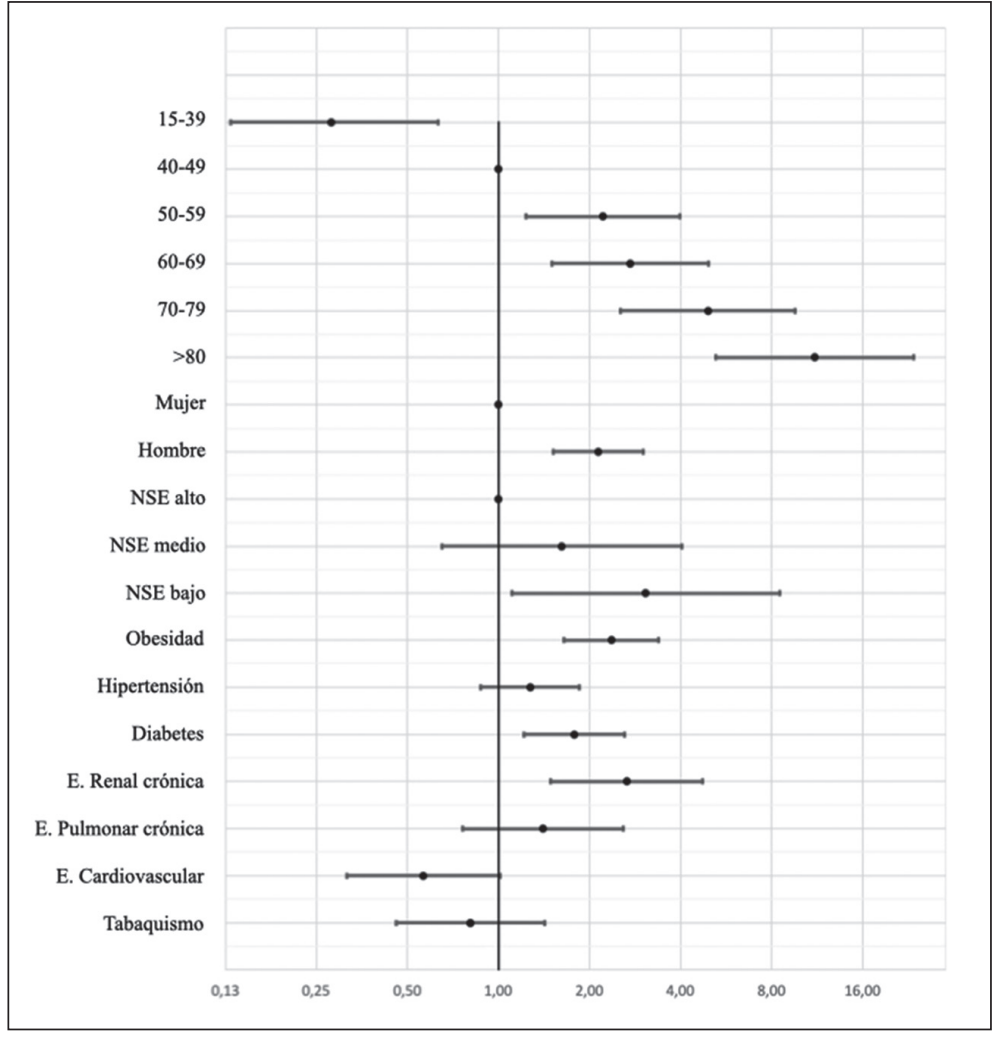

Figura 2. Forest plot de Odds ratio e intervalos de confianza 95\% del modelo completamente ajustado para cada categoría de edad, sexo, nivel socioeconómico y comorbilidades. 


\begin{abstract}
Tabla 2. Odds ratio e intervalo de confianza 95\% para outcome severo de COVID-19 (cuidados críticos o muerte) tanto para el modelo ajustado sólo por sexo y edad, como para el modelo totalmente ajustado
\end{abstract}

\begin{tabular}{|llcc|}
\hline & & Ajustado por sexo y edad & Totalmente ajustado \\
Edad & $15-39$ & $0,20(0,09-0,43)$ & $0,28(0,13-0,63)$ \\
& $40-49$ & $1,00($ ref $)$ & $1,00($ ref $)$ \\
& $50-59$ & $2,36(1,36-4,09)$ & $2,22(1,23-3,99)$ \\
& $60-69$ & $3,85(2,24-6,63)$ & $2,73(1,51-4,94)$ \\
& $70-79$ & $6,95(3,81-12,69)$ & $1,92(2,54-9,55)$ \\
Sexo & $>80$ & $13,04(6,57-25,88)$ & $1,08(5,22-23,53)$ \\
& Mujer & $1,00($ ref $)$ & $2,13(1,52-3,00)$ \\
Nivel socio- & Hombre & $1,92(1,39-2,64)$ & $1,00($ ref $)$ \\
económico & Alto & $1,00($ ref $)$ & $1,62(0,65-4,04)$ \\
& Medio & $2,01(0,82-4,93)$ & $3,07(1,11-8,52)$ \\
Comorbilidades & $2,36(1,65-3,39)$ \\
& Bajo & $4,13(1,52-11,25)$ & $1,27(0,88-1,85)$ \\
& Obesidad & $2,78(1,99-3,89)$ & $1,78(1,22-2,61)$ \\
& Hipertensión & $1,82(1,30-2,55)$ & $2,65(1,49-4,73)$ \\
& Diabetes & $2,48(1,76-3,51)$ & $1,40(0,76-2,58)$ \\
& Enfermedad renal crónica & $2,90(1,67-5,03)$ & $0,57(0,32-1,01)$ \\
& Enfermedad pulmonar crónica & $1,49(0,85-2,60)$ & $0,81(0,46-1,42)$ \\
\hline
\end{tabular}

\section{Resultados principales}

De todas las variables analizadas en este estudio, la edad avanzada y el nivel socioeconómico bajo fueron las variables más fuertemente asociadas con un resultado de COVID-19 severo, considerado como muerte o cuidados críticos. Se observó un claro gradiente para la edad, donde las personas de 80 años o más tenían 11 veces más chance de cuadro severo que el grupo de 40-49 años (OR totalmente ajustado 11,08; IC del 95\%: 5,22-23,53). El nivel socioeconómico bajo mostró chance de cuadro severo más de tres veces mayores que el nivel socioeconómico alto (totalmente ajustado 3,07; IC 95\% 1,11-8,52).

El sexo masculino también se asoció fuertemente con severidad, con una chance más de dos veces mayor que las mujeres (totalmente ajustado 2,13; 95\% CI 1,52-3,00).

La enfermedad renal crónica, la obesidad y la diabetes se asociaron con una mayor gravedad en orden descendente. No se encontró asociación estadísticamente significativa para hipertensión, enfermedad pulmonar crónica, enfermedad cardiovascular o tabaquismo. Todos los resultados del modelo ajustados por edad y sexo y completamente ajustados y sus intervalos de confianza se presentan en la Tabla 2.

\section{Discusión}

\section{Resultados clave}

La edad avanzada y el sexo masculino se asociaron fuertemente con un mal pronóstico de COVID-19 consistente con otros estudios ${ }^{4,5,7-9,17}$. Es de destacar que en los mayores de 60 años el número de defunciones por grupo de edad supera al número de pacientes ingresados en UCI con un gradiente que aumenta con la edad (Tabla 1). Por lo tanto, en mayores de 60 años, la muerte es más probable fuera de una UCI que adentro, que podría ser explicado por un agravamiento repentino, por falta de disponibilidad de camas críticas, o decisión de limitar el esfuerzo terapéutico.

El nivel socioeconómico bajo resultó ser el factor más importante asociado con el resultado severo después de la edad. Esta asociación se observa sólo con respecto al nivel socioeconómico bajo, 
pero no se evidencia entre el nivel socioeconómico medio y el alto. Esto podría explicarse por el hecho de que la categoría media es mucho más heterogénea dado que incorpora asegurados de salud de las secciones $\mathrm{B}, \mathrm{C}$ y D, probablemente incluyendo personas de altos ingresos que no han migrado a un seguro privado. Esta heterogeneidad podría ocultar el gradiente de riesgo existente según el nivel socioeconómico que se ha informado previamente en una cohorte del Reino Unido9. Múltiples revisiones internacionales como las de Espinosa et al. ${ }^{4}(\mathrm{n}=89,238)$, Gold et al. ${ }^{5}(\mathrm{n}=29,096)$, Abate et al. ${ }^{6}(n=24.998)$, Fang et al. $(n=8.140)^{7}$ y Noor et al. $(\mathrm{n}=122,191)^{8}$ no toman en cuenta las variables socioeconómicas en su análisis multivariado, por lo que se corre el riesgo de presentar un efecto de confusión de comorbilidades en el pronóstico, dado que las comorbilidades también son más prevalentes en los grupos socioeconómicos más bajos (siendo un camino abierto por la puerta trasera $)^{5}$. A la luz de estos hallazgos es fundamental considerar el nivel socioeconómico en el pronóstico y hacerlo parte de la discusión de la gestión académica y sanitaria de esta pandemia, siendo un punto crítico el desafío futuro de disminuir las inequidades en nuestro país.

La enfermedad renal crónica fue la comorbilidad medida más fuertemente asociada con un cuadro severo. Esta asociación es consistente con la evidencia disponible ${ }^{8,9}$ y la magnitud de esta asociación también es consistente con la descrita por otros autores que han reportado que la ERC contribuyó en gran medida al desenlace de la muerte $^{7,18}$. Incluso ha sido señalado por algunos autores como un factor de riesgo clave ${ }^{19}$. En consecuencia, estos pacientes merecen una atención especial por su alto riesgo de cuadro severo.

La obesidad también ha sido descrita como un factor de riesgo y pronóstico importante para el COVID-19 adverso, con mayores necesidades de tratamiento médico ${ }^{20-22}$ avanzado principalmente en hombres jóvenes ${ }^{23}$. Los resultados de este estudio destacan la importancia de la obesidad independientemente del efecto mediador que producen otras enfermedades como la diabetes o la enfermedad renal crónica, de acuerdo con la literatura ${ }^{24}$.

La diabetes también mostró un papel importante en la evolución desfavorable independientemente de otras comorbilidades. Este efecto se explica independientemente de la obesidad ya que el modelo elimina los efectos de confusión. Se requiere completar esta información diferenciando por el nivel de compensación de diabetes, en consonancia con otros estudios que han mostrado una gradiente según el nivel de HbAlc9. Esta comorbilidad tiene un gran impacto especialmente en los países de bajos ingresos dado que tienen la mayor prevalencia de diabetes ${ }^{25}$. Es necesario considerar las diferencias en la prevalencia de factores de riesgo en diferentes áreas geográficas y puede explicar en parte las diferencias en morbilidad y mortalidad por COVID-19 en diferentes partes del mundo ${ }^{26}$.

En este estudio no se encontró asociación entre hipertensión o tabaquismo activo concordante con otros estudios que han mostrado asociaciones inexistentes o débiles ${ }^{7,9}$. La enfermedad pulmonar crónica y la enfermedad cardiovascular también mostraron una asociación nula, lo que podría explicarse en parte por la dificultad para establecer los criterios diagnósticos desde la HEC dada la agregación de múltiples diagnósticos que podrían haber sido infrarreportados en el sistema.

\section{Fortalezas y limitaciones}

Entre las fortalezas destaca la utilización exclusiva de casos confirmados incluyendo casos hospitalizados y ambulatorios. La consideración del nivel socioeconómico permite incorporar una variable que no ha sido suficientemente atendida y adquiere particular relevancia en el contexto social que está viviendo nuestro país. Desde el punto de vista estadístico las fortalezas son el tamaño de la cohorte que permitió un poder estadístico suficiente para obtener resultados significativos con un intervalo de confianza de $95 \%$ y la presentación desagregada de los datos para uso por otros investigadores. Las principales limitaciones son la falta de estandarización de los diagnósticos por utilizar registros clínicos de base secundaria, mismo problema que presenta el sistema EPIVIGILA dónde las patologías son reportadas por quién ingresa la notificación al sistema. Esto además implicó no poder contar con la intensidad de exposición a las comorbilidades (como nivel de obesidad, etapa de enfermedad renal crónica y nivel de compensación de diabetes) que hubiera permitido encontrar gradientes de exposición. Por otro lado, las condiciones sociales que determinan vulnerabilidad son multidimensionales y en este estudio solo se consideró ingreso formal medido en forma indi- 
recta a través del seguro de salud (previsión). Podría interpretarse el resultado más explícito como establecer que pertenecer al tramo A de FONASA es un factor de riesgo de mal pronóstico, y que a juicio de los autores sería representación indirecta del nivel socioeconómico. Este estudio muestra los resultados de una cohorte de un hospital de Chile que a su vez son consistentes con la literatura internacional en la materia. Si bien el diseño muestral no permite generalizar resultados a todo el país, parece razonable considerarlo.

\section{Implicaciones futuras}

La población chilena de bajo nivel socioeconómico (entendidos como pertenecientes a tramo A de FONASA) deben ser consideradas prioritarias en la distribución de las vacunas disponibles. Las actuales recomendaciones de priorización en Chile $^{27}$ no consideran el nivel socioeconómico ni algún símil, invisibilizando el rol clave que tienen los determinantes sociales en el nivel de salud, que ha sido mostrado en estudios internacionales y ratificado este estudio en Chile. En el contexto político coyuntural que enfrenta Chile previo a la redacción de una nueva constitución, es fundamental poner sobre la mesa las implicancias que tienen los determinantes sociales en el nivel de salud de las poblaciones desde un enfoque de derechos humanos.

Agradecimientos: Al director del Hospital el Carmen, Dr. Juan Kehr quien facilitó la información para realizar el estudio. Al Dr. Daniel Pincheira-Donoso por los aportes prácticos desde la mirada de la ciencia. Al traductor Keith Carr para el trabajo en la versión en inglés, a Corina Navarro por su apoyo en trabajo administrativos y a Camila Grandon por su apoyo en la recolección de datos. Finalmente, a todas las personas que han dado su vida y sus familias.

\section{Referencias}

1. Departamento de Epidemiología M de S de C. Informe epidemiológico $\mathrm{N}^{\circ}$ 87, Enfermedad por SARS-CoV-2 (COVID-19). 2021. Available from: https://www.minsal. cl/wp-content/uploads/2021/01/Informe-Epidemiologico-87.pdf

2. Verity R, Okell LC, Dorigatti I, Winskill P, Whittaker
C, Imai N, et al. Estimates of the severity of coronavirus disease 2019: a model-based analysis. Lancet Infect Dis. 2020; 20 (6): 669-77.

3. Wu Z, McGoogan JM. Characteristics of and Important Lessons from the Coronavirus Disease 2019 (COVID-19) Outbreak in China: Summary of a Report of 72314 Cases from the Chinese Center for Disease Control and Prevention. JAMA - J Am Med Assoc. 2020; 323 (13): 1239-42.

4. Espinosa OA, Zanetti A dos S, Antunes EF, Longhi FG, Matos TA de, Battaglini PF. Prevalence of comorbidities in patients and mortality cases affected by SARS-CoV2: a systematic review and meta-analysis. Rev Inst Med Trop Sao Paulo. 2020; 62 (1): 1-17.

5. Gold MS, Sehayek D, Gabrielli S, Zhang X, McCusker C, Ben-Shoshan M. COVID-19 and comorbidities: a systematic review and meta-analysis. Postgrad Med. 2020; 00 (00): 1-7.

6. Abate SM, Ali SA, Mantfardo B, Basu B. Rate of intensive care unit admission and outcomes among patients with coronavirus: A systematic review and Meta-analysis. PLoS One. 2020; 15 (7 July): 1-19.

7. Fang X, Li S, Yu H, Wang P, Zhang Y, Chen Z, et al. Epidemiological, comorbidity factors with severity and prognosis of COVID-19: A systematic review and meta-analysis. Aging (Albany NY). 2020; 12 (13): 12493503.

8. Musharrat F, Momin N. Prevalence and Associated Risk Factors of Mortality Among COVID-19 Patients: A Meta - Analysis. J Community Health. 2020;(0123456789).

9. Williamson EJ, Walker AJ, Bhaskaran K, Bacon S, Bates C, Morton CE, et al. Factors associated with COVID-19-related death using OpenSAFELY. Nature. 2020; 584 (7821): 430-6.

10. Deng G, Yin M, Chen X, Zeng F. Clinical determinants for fatality of 44,672 patients with COVID-19. Crit Care. 2020; 24 (1): 1-3.

11. Mena GE, Martínez PP, Mahmud AS, Marquet PA, Buckee CO, Santillana M. Socioeconomic status determines COVID-19 incidence and related mortality in Santiago, Chile. Science (80-) [Internet]. 2021 Apr 27 [cited 2021 May 6];eabg5298. Available from: https:// science.sciencemag.org/content/sci/early/2021/04/26/ science.abg5298.full.pdf.

12. Tan AX, Hinman JA, Abdel Magid HS, Nelson LM, Odden MC. Association Between Income Inequality and County-Level COVID-19 Cases and Deaths in the US. JAMA Netw open [Internet]. 2021; 4 (5): e218799. Available from: http://www.ncbi.nlm.nih.gov/pubmed/33938935. 
13. Pan American Health Organization. PAHO COVID-19 RESPONSE. COVID-19 Information System for the Region of the Americas .

14. Yang J, Zheng Y, Gou X, Pu K, Chen Z, Guo Q, et al. Prevalence of comorbidities and its effects in patients infected with SARS-CoV-2: a systematic review and meta-analysis. Int J Infect Dis. 2020; 94 (April): 91-5.

15. Cifuentes P. Perfil de edad e ingresos, según previsión en salud. Rev Chil Salud Pública. 2015; 19 (3):315.

16. Fondo Nacional de Salud. FONASA. Tramos 2021. Available from: https://www.fonasa.cl/sites/fonasa/tramos

17. Deng G, Yin $M$, Chen X, Zeng F. Clinical determinants for fatality of 44 , 672 patients with COVID-19. 2020;13.

18. Oyelade T, Alqahtani J, Canciani G. Prognosis of COVID-19 in Patients with Liver and Kidney Diseases: An Early Systematic Review and Meta-Analysis. Trop Med Infect Dis. 2020; 5 (2): 80.

19. Gansevoort RT, Hilbrands LB. CKD is a key risk factor for COVID-19 mortality. Nature Reviews Nephrology. Nature Research; 2020. p. 1-2.

20. Cai Q, Chen F, Wang T, Luo F, Liu X, Wu Q, et al. Obesity and COVID-19 Severity in a Designated Hospital in Shenzhen, China. Diabetes Care [Internet]. 2020; 43 (7): 1392-8. Available from: http://care.diabetesjournals.org/ lookup/doi/10.2337/dc20-0576.

21. Wang T. The Lancet Infectious Diseases Obesity and COVID-19 severity in a designated hospital in Shenzhen, China. 2020;(202002073000001).
22. Simonnet A, Chetboun M, Poissy J, Raverdy V, Noulette J, Duhamel A, et al. High Prevalence of Obesity in Severe Acute Respiratory Syndrome Coronavirus-2 (SARSCoV-2) Requiring Invasive Mechanical Ventilation. Obesity. 2020; 28 (7): 1195-9.

23. Tartof SY, Qian L, Hong V, Wei R, Nadjafi RF, Fischer $\mathrm{H}$, et al. Obesity and Mortality Among Patients Diagnosed With COVID-19: Results From an Integrated Health Care Organization. Ann Intern Med. 2020.

24. Tamara A, Tahapary DL. Obesity as a predictor for a poor prognosis of COVID-19: A systematic review. Diabetes Metab Syndr Clin Res Rev. 2020; 14 (4): 655-9.

25. Dagenais GR, Gerstein HC, Zhang X, McQueen M, Lear S, Lopez-Jaramillo $\mathrm{P}$, et al. Variations in diabetes prevalence in low-, middle-, and high-income countries: Results from the prospective urban and rural epidemiological study. Diabetes Care. 2016; 39 (5): 780-7.

26. Clark A, Jit M, Warren-Gash C, Guthrie B, Wang HHX, Mercer SW, et al. Global, regional, and national estimates of the population at increased risk of severe COVID-19 due to underlying health conditions in 2020: a modelling study. Lancet Glob Heal. 2020; 8 (8): e100317.

27. CAVEI. Pronunciamiento interino del CAVEI sobre grupos objetivo de vacunar contra SARS-CoV-2 [Internet]. 2020. Available from: https://vacunas.minsal.cl/ wpcontent/uploads/2020/07/CAVEI_Pronunciamiento-Interino-Grupos-VacunaciónCOVID19_30JUN2020. pdf\%0A\%0A\%0A. 